NP28 (continued)

Objective: Produce final versions of two validated obesity risk assessment tools for Spanish speaking parents; assess feasibility of these tools to the child's physician; develop and validate a diet quality tool; and determine feasibility of an EFNEP intervention in a medical clinic.

Description: Protocol development, enrollment and data collection commenced in year 2 to validate the Spanish obesity risk assessment tools using: parental surveys; 24-hour dietary, sleep, activity logs; anthropometric and biomarker measures; and mealtime video recordings. The 23-item diet quality tool in English and Spanish was tested for face and content validity. The EFNEP intervention protocol, education materials including motivational modeling were finalized, a bilingual educator was trained, and an information kiosk is ready for pilot testing.

Evaluation: To date, recruitment has focused on eight Head Start sites with a total of 110 Spanish speaking parent-child pairs enrolled with a sub-sample $(n=24)$ completing mealtime videotaping. Preliminary analyses of the glucose and lipid profiles show that the child metabolic data are positively associated with BMI percentile. Physicians $(n=38)$ from four medical clinics have generated 198 patient referrals for the EFNEP intervention. Parents $(n=39)$ have been enrolled in the intervention which required 584 phone calls and 368 texts. Sixty-five percent of parents stated that the physician referral was an important reason for enrolling and most parents and physicians report value from the intervention.

Conclusions and Implications: Validation of two pediatric obesity risk assessment tools and one diet quality tool are underway. An EFNEP intervention is demonstrating feasibility at a medical clinic site yet challenges remain in garnering physician referrals. Outcomes may contribute to the identification of young children more likely to become obese.

Funding: 2015-68001-23280

\section{NP29 Development of a Medical Center Kiosk to Promote Pediatric Obesity Risk Reduction}

Mical Kay Shilts, PhD, California State University, Sacramento; Misty Reed, MA, MFA, University of California, Davis; Larissa Leavens, BA; Lenna Ontai, $P h D$; Louise Lanoue, PhD; Dennis Styne, MD;

MarilynS. Townsend,PhD, RD, mstownsend@ucdavis.edu, University of California, Davis, 1 Shields Avenue, Davis, CA 95616

Background: To assist with pediatric obesity risk reduction, pediatricians have partnered with the Expanded Food and Nutrition Education Program (EFNEP). Parents are referred by pediatricians to receive nutrition education in the medical clinic site provided by EFNEP.
Objective: Development of an education kiosk to be placed in the clinic waiting room to facilitate pediatric obesity risk communication between parents of pediatric patients and pediatricians and increase referrals to the EFNEP intervention.

Description: Select kiosk content. Conduct formal planning meetings with researchers, medical clinic and IT (Information Technology) staff to determine type of kiosk plus location and content output (directly to patient or via medical record). Design and code kiosk program based on content selected and medical staff priorities.

Evaluation: Content of kiosk was based on previous longitudinal validation results of two pediatric obesity risk assessment tools, Healthy Kids and My Child at Mealtime. Based on three meetings with clinic staff and physicians, and two meetings with IT staff, a small scale, mobile iPad kiosk with attached receipt style printer and location in the clinic were determined. The kiosk programming generated tailored pediatric obesity prevention messages based on a 21-item survey. The tailored printout encourages the parent to discuss messages with their pediatrician and the EFNEP intervention offered at the clinic.

Conclusion and Implications: Development of a medical center kiosk to facilitate pediatric obesity risk reduction requires collaboration between content experts, clinic staff, IT personal, design and programming experts. Printing nutrition messages via iPad kiosk proved challenging due to medical center Wi-Fi connectivity and security issues. The kiosk will be pilot tested with parents in spring 2017.

Funding: 2015-68001-23280

\section{NP30 Process for Validating My Child at Mealtime: Assessing Parent Mealtime Behavior in Spanish Speaking Families of Preschoolers}

Lenna Ontai, PhD, University of California, Davis; Karina Diaz Rios, $P h D$, University of California, Merced; Mical Kay Shilts, PhD, California State University, Sacramento; Louise Lanoue, $P h D$, University of California, Davis; Christine Davidson, BA; Gloria Zavala, BAS; Maria Espinoza, BS; Marilyn Townsend, $P h D, R D$, mstownsend@ucdavis.edu, University of California, Davis, 1 Shields Avenue, Davis, CA 95616

Objective: Describe the process for validating the Spanish language My Child at Mealtime (MCMT) tool which assesses parent feeding styles in low-literacy, Spanishspeaking parents of preschool children.

Description: Parent feeding styles are considered effective targets for obesity prevention programs. Accurate measurement of feeding styles is a challenge for federally-funded, group-based nutrition education programs that rely on self-assessments with low literacy demands. Higher rates of obesity in Hispanic and Latino children warrant the development of valid self-assessment tools 